\title{
Structural Characterization of Microcystins by LC/MS/MS under Ion Trap Conditions
}

\author{
Tsuyoshi Mayumi, Hajime Kato, Susumu Imanishi, Yoshito Kawasaki, \\ Masateru Hasegawa, Ken-ichi Harada \\ This article is dedicated in memory of Professor Kenneth L. Rinehart at the University of \\ Illinois
}

Received: May 16, 2006 / Accepted: November 2, 2006

(C) Japan Antibiotics Research Association

\begin{abstract}
LC/MS/MS under ion trap conditions was used to analyze microcystins produced by cyanobacteria. Tandem mass spectrometry using $\mathrm{MS}^{2}$ was quite effective since ions arising from cleavage at a peptide bond provide useful sequence information. The fragmentation was confirmed by a shifting technique using structurally-related microcystins and the resulting fragmentation pattern was different from those determined by triple stage MS/MS and four sector MS/MS. Analysis of a mixture of microcystins in a bloom sample was successfully performed and two new microcystins were identified by LC/MS/MS under ion trap conditions. Thus, LC/MS/MS under ion trap conditions is effective for the structural characterization of microcystins.
\end{abstract}

Keywords LC/MS/MS under ion trap conditions, cyclic peptide, microcystin, structural analysis, sequence information, fragmentation pattern

\section{Introduction}

Cyclic peptides have been often found and isolated from microorganisms, cyanobacteria, fungi and marine sponges as bioactive secondary metabolites, and some of them have been developed as medicines. For instance, cyclic peptide antibiotics such as bacitracin [1], colistin [2], vancomycin [3] and micafungin [4], are widely used for medical treatments. Moreover, cyclosporin [5] is an immunosuppressive agent, which has been used for organ transplant rejection or bone marrow [6].

For the structure determination of cyclic peptides, the following information is required: structures, absolute configurations and sequence of constituent amino acids. Although partial acid hydrolysis had been used for the structure determination of these cyclic peptides, it has been currently performed by instrumental methods such as 2DNMR (two dimensional nuclear magnetic resonance) and MS/MS (tandem mass spectrometry) techniques. However, these techniques have the following inherent drawbacks: overlapped signals derived from carbonyl carbons and $\alpha$ hydrogens of the constituent amino acids and nonreproducible fragmentation by tandem mass spectrometry with high- (four sector MS/MS) or low- (triple stage MS/MS) energetic CID (collision activation dissociation). Therefore, a more reliable and effective methodology has been eagerly required for cyclic peptides [7].

Microcystins (Fig. 1), a family of cyclic heptapeptides isolated from cyanobacteria, show potent inhibition of protein phosphatases 1 and 2A [8]. Their structures were determined in 1984 [9], and 67 related compounds have been isolated since then [10]. Although triple stage MS/MS
K. Harada (Corresponding author): Graduate School of Environmental and Human Science \& Faculty of Pharmacy, Meijo University, Tempaku, Nagoya 468-8503, Japan,

E-mail: kiharada@ccmfs.meijo-u.ac.jp
T. Mayumi, H. Kato, S. Imanishi, Y. Kawasaki, M. Hasegawa: Graduate School of Environmental and Human Science, Meijo University, Tempaku, Nagoya 468-8503, Japan 


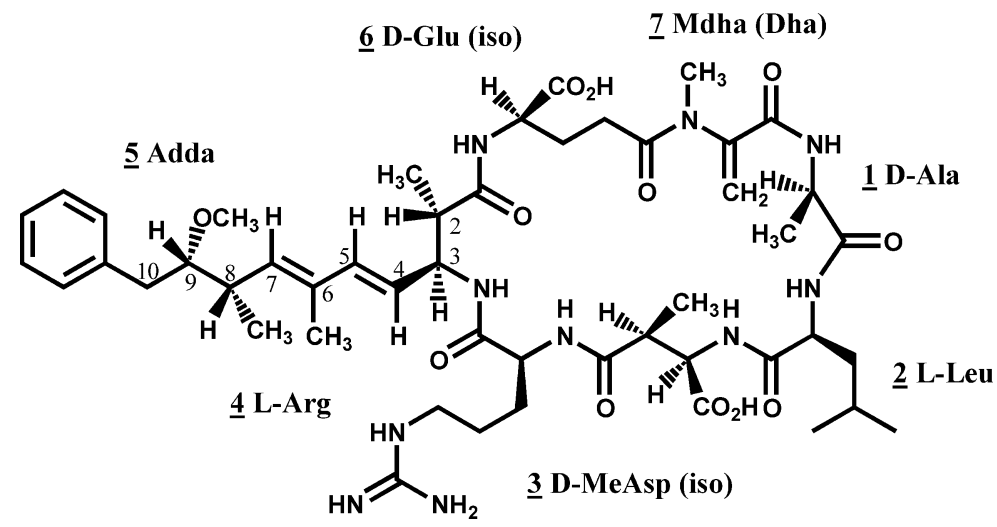

\begin{tabular}{|c|c|c|c|c|c|c|c|c|}
\hline Microcystins (abbreviation) & M.W. & 1 & 2 & 3 & 4 & 5 & 6 & 7 \\
\hline Microcystin-LR (MCLR) & 994 & D-Ala & L-Leu & D-MeAsp & L-Arg & Adda & L-Glu & Mdha \\
\hline Microcystin-YR (MCYR) & 1044 & D-Ala & $\underline{\mathrm{L}-T \mathrm{y} r}$ & D-MeAsp & L-Arg & Adda & L-Glu & Mdha \\
\hline Microcystin-RR (MCRR) & 1037 & D-Ala & $\underline{L}-\operatorname{Arg}$ & D-MeAsp & L-Arg & Adda & L-Glu & Mdha \\
\hline Microcystin-LF (MCLF) & 985 & D-Ala & L-Leu & D-MeAsp & $\underline{\text { L-Phe }}$ & Adda & L-Glu & Mdha \\
\hline Microcystin-LW (MCLW) & 1025 & D-Ala & L-Leu & D-MeAsp & $\underline{\underline{L}-\operatorname{Trp}}$ & Adda & L-Glu & Mdha \\
\hline 3-Desmethylmicrocystin-LR (3-Des-MCLR) & 980 & D-Ala & L-Leu & $\underline{\mathrm{D}-A s p}$ & L-Arg & Adda & L-Glu & Mdha \\
\hline 7-Desmethylmicrocystin-LR (7-Des-MCLR) & 980 & D-Ala & L-Leu & D-MeAsp & L-Arg & Adda & L-Glu & $\underline{\text { Dha }}$ \\
\hline Dihydromicrocystin-LR (Dihydro-MCLR) & 996 & D-Ala & L-Leu & D-MeAsp & L-Arg & Adda & L-Glu & $\underline{\text { MeAla }}$ \\
\hline
\end{tabular}

Adda: [(2S,3S,8S,9S)-3 amino-9-methoxy-10-phenyl-2,6,8-trimethyldeca-4(E),6(E)-dienoic acid, Mdha: N-methyldehydroalanine, Dha: Dehydroalanine, MeAsp: $\beta$-erythro-methyl-D-Asp, MeAla: N-methylalanine.

Fig. 1 Structures of microcystin LR (MCLR) and related compounds.

and four sector MS/MS were applied to characterize the microcystin structures [11 14], the resulting fragmentations were complicated and it was difficult to use them for the structural characterization of unknown microcystins. Recently, LC/MS/MS (liquid chromatography/ tandem mass spectrometry) under ion trap conditions has been applied to the structure elucidation of various compounds with low to high molecular weights, and mainly to the amino acid sequence determination in the proteomics study and in vivo or in vitro metabolite screening during the process of drug development [15 17]. From LC/MS/MS under ion trap conditions, it is possible to acquire much information with a small amount of sample and then use the $\mathrm{MS}^{\mathrm{n}}$ technique. For the $\mathrm{MS}^{2}$ and $\mathrm{MS}^{3}$ analyses of microcystin using LC/MS/MS under ion trap conditions, the resulting fragmentation pattern was simpler than those with other MS/MS methods. Poon et al. reported good results for the analysis of microcystins using this system. However, the elucidation of the fragmentation for microcystin-LR (MCLR) was not enough to apply it to the structure characterization of unknown microcystins [18]. Moreover, although Deihnelt et al. analyzed various microcystins using ion trap Fourier transform ion cyclotron, this equipment has not been widely used and not been a standard tool [10]. Therefore, we have made an attempt to apply LC/MS/MS under ion trap conditions to extend its applicability for the structure elucidation of unknown microcystins.

In the present study, we confirmed the fragmentation pattern of microcystins using LC/MS/MS under ion trap conditions, which was used for the structure characterization of non-isolated microcystins after simple extraction from a bloom sample [19].

\section{Materials and Methods}

\section{Chemicals and Reagents}

Microcystins, -LR, -YR, -RR, -LW, -LF, 3-desmethyl-LR and 7-desmethyl-LR were isolated and purified by silica-gel chromatography and gel filtration chromatography from the lyophilized cells of cyanobacteria collected in various sources or fermented strains [20]. Dihydromicrocystin-LR was prepared by the reduction of microcystin-LR with $\mathrm{NaBH}_{4}$ [21]. These microcystins were then dissolved in methanol $(10 \mu \mathrm{g} / \mathrm{ml})$. Each injection volume was $5 \mu \mathrm{l}$ for 
the analysis. A TSK-gel Super ODS column, $2.0 \times 100 \mathrm{~mm}$ (Tosoh Co. Ltd., Tokyo, Japan) was used for the fractionation of the microcystins. Methanol and formic acid were purchased from Nacalai tesque Co. Ltd. (Kyoto, Japan). Pure water was obtained using the E-pure distillation and ion exchange system (Barnstead, Germany). A 5\% acetic acid solution was used for the extraction of the microcystins from lyophilized cells collected from Lake Suwa, Japan. The Sep-pak cartridge (ODS, $500 \mathrm{mg}$ ) used for the extraction of microcystin from the lyophilized cells was purchased from Waters Co., Ltd. (MA, USA).

\section{Instrumentation}

The Agilent Technologies (Waldbronn, Germany) 1100 series was used as the HPLC system that consisted of binary pump, column compartment module, degasser, and UV-VIS detector. The LC/MS/MS system was a Thermo Electron LCQ Deca XP plus (San Jose, CA, USA) equipped with an electrospray ionization (ESI) interface.

\section{LC/MS Conditions}

The chromatographic system consisted of an ODS column with the mobile phase of $\mathrm{A}$ : water and $\mathrm{B}$ : methanol containing $0.1 \%$ formic acid. The LC gradient elution conditions were initially $40 \%$ B to $90 \%$ B over 30 minutes. The flow rate was set at $0.2 \mathrm{ml} /$ minute. The $\mathrm{MS}^{2}$ analysis was used in the product ion scan in centroid mode. In the $\mathrm{MS}^{2}$ mode, detection was in the positive ionization mode. The source voltage was $5.0 \mathrm{kV}$. The temperature of the heated capillary was $300^{\circ} \mathrm{C}$. Full-scan mass spectra were acquired over $\mathrm{m} / z \quad 400 \sim 1500$ within 1.0 second. Helium was used as the collision gas in the range of $1 \sim 2$ mTorr. The protonated molecule was activated at $35 \%$ different collision energy level.

\section{Extraction of Microcystins}

Extraction was carried out using the established method as follows [19]: a $100 \mathrm{mg}$ sample of lyophilized cells of cyanobacteria collected from Lake Suwa was extracted with $10 \mathrm{ml}$ of a $5 \%$ acetic acid solution for 30 minutes, and the resulting extract was centrifuged at $3000 \mathrm{~g}$ for 10 minutes. This extraction was repeated 3 times. After the filtration, the supernatant was applied to an ODS cartridge, and the cartridge was washed with $5 \mathrm{ml}$ of water, followed by $5 \mathrm{ml}$ of $20 \%$ methanol. The desired compounds were eluted with $5 \mathrm{ml}$ of $90 \%$ methanol and then $5 \mathrm{ml}$ of methanol. Both fractions containing the microcystins were collected and evaporated to dryness, and the residue was dissolved in methanol.

\section{Results}

\section{Fragmentation Pattern of Arg Containing Microcystin}

The general structure of microcystin is cyclo (-D-Ala ${ }^{1}-\mathrm{R}_{2}{ }^{2}$ D-MeAsp ${ }^{3}-\mathrm{R}_{4}{ }^{4}$-Adda ${ }^{5}$-D-Glu ${ }^{6}-\mathrm{Mdha}^{7}$-), where $\mathrm{R}_{2}$ and $\mathrm{R}_{4}$ are the two variable L-amino acids, and their abbreviations are used as the suffix of the amino acid name (Fig. 1). Adda, Mdha and MeAsp denote $(2 S, 3 S, 8 S, 9 S)$-3-amino-9methoxy-10-phenyl-2,6,8-trimethyldeca-4(E),6(E)-dienoic acid [22], $N$-methyldehydroalanine and $\beta$-methylaspartic acid, respectively. MCLR (Fig. 1, M.W. 994) is the most popular member found among the microcystins, where its two L-amino acid positions are Leu as $\mathrm{R}_{2}$ and Arg as $\mathrm{R}_{4}$. The $\mathrm{MS}^{2}$ spectrum of the protonated molecule $(\mathrm{M}+\mathrm{H})^{+}$at $\mathrm{m} / \mathrm{z} 995$ of MCLR obtained from the LC/MS/MS on ion trap analysis is shown in Fig. 2.

The prominent fragment ions at $\mathrm{m} / \mathrm{z} 866$ (Ion $a, e$ ), 728 (Ion g), 710 (Ion $g^{\prime}$ ), 682 (Ion b), 599 (Ion c), 570 (Ion f'), $553($ Ion $f)$ and $470($ Ion $d, h)$ were observed together with some ions in the higher mass region of the $\mathrm{MS}^{2}$ spectrum. These ions are generated by the cleavage of peptide bonds, because the mass numbers of the ions correspond to the difference between the precursor ion $(\mathrm{m} / \mathrm{z} 995)$ and the liberated peptide moieties. Generally, these ions must be formed by the two-bond fissions of the precursor ion, in which the initial fission of the cyclic structure to a linearized one and subsequent fission(s) at the peptide bonds are included. For the MCLR, the following peptide bonds, Arg-MeAsp, MeAsp-Leu, Ala-Mdha and MdhaGlu, were initially cleaved as shown in Fig. 3. The resulting fragment ions were classified into four groups: (A) Ions $a \sim d$ by the cleavage at Arg-MeAsp, (B) Ion e, $f^{\prime}$ by the cleavage at Mdha-Glu, (C) Ions $g$ and $g^{\prime}$ by the cleavage at MeAsp-Leu and (D) Ion $h$ by the cleavage Ala-Mdha. As shown in Fig. 3, it was found that the observed ions always contain the Arg moiety.

In order to rationalize the fragmentation of MCLR using the LC/MS/MS under ion trap conditions mentioned above, we compared the fragmentation of MCLR with those of three related microcystins that are structurally, but slightly different: dihydromicrocystin-LR [21] (dihydro-MCLR, M.W. 996) contains MeAla ( $N$-methylalanine) instead of Mdha, microcystin-YR (MCYR, M.W. 1044) has Tyr replaced by Leu in MCLR, and 3-desmethymicrocystin-LR (3-des-MCLR, M.W. 980) is a desmethylated analogue of MCLR at the 3 position (Fig. 1). The predominant fragment ions observed in the $\mathrm{MS}^{2}$ spectra of the related microcystins are summarized in Table 1 . This is a shifting technique [23], and these ions are classified into two groups, the shifted or non-shifted groups as follows: in the 


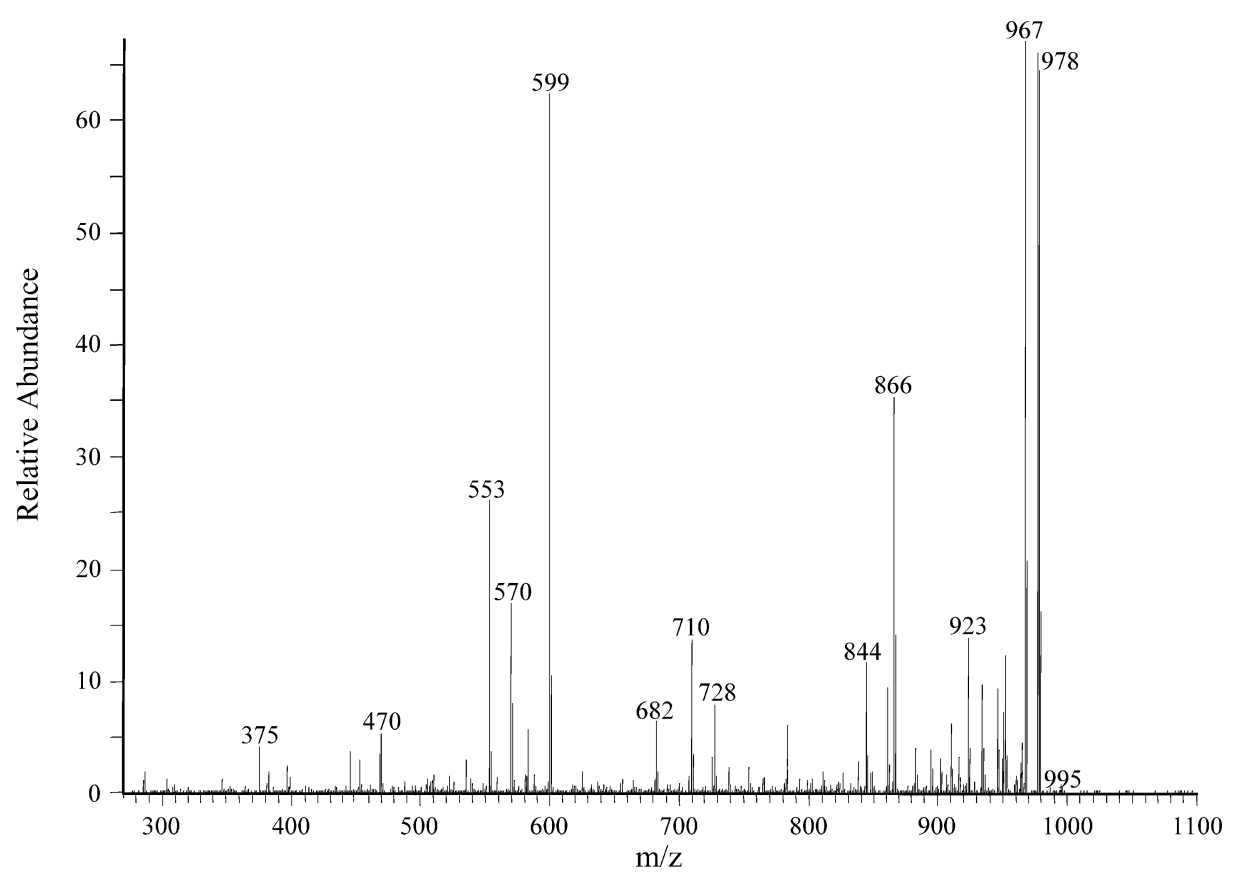

Fig. $2 M S^{2}$ spectrum of $M C L R$.

\begin{tabular}{|c|c|c|c|c}
\cline { 2 - 4 } \multicolumn{1}{r|}{} & Glu & \multicolumn{2}{c|}{ Mdha } & \\
\hline Adda & + & Ala & MCLR \\
\hline & Arg & MeAsp & Leu & M \\
\hline
\end{tabular}

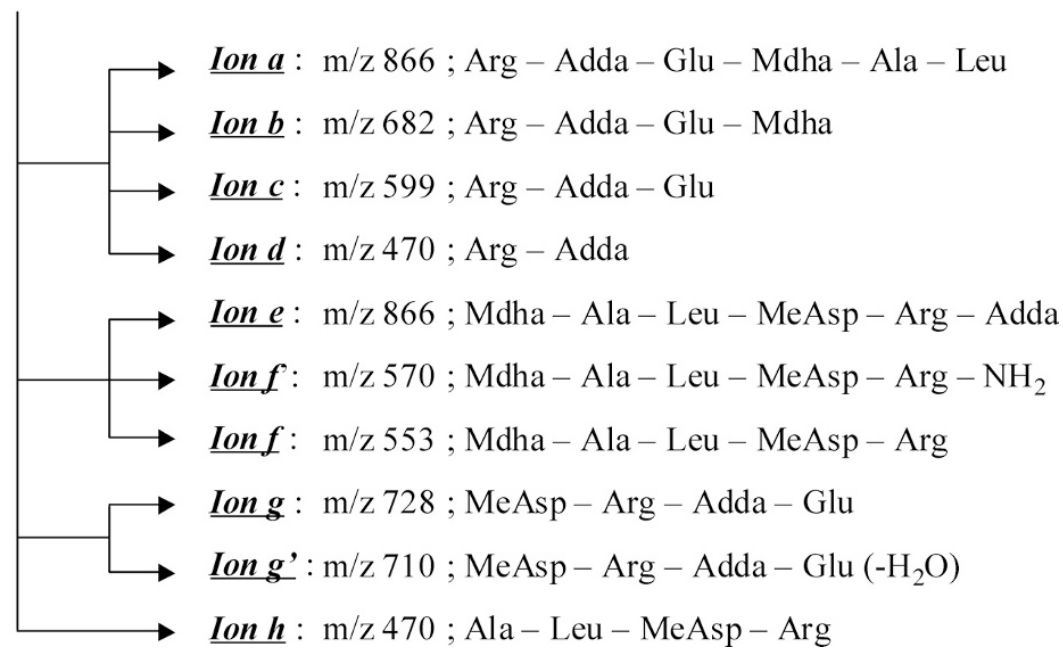

Fig. 3 Fragmentation scheme of MCLR using LC/MS/MS under ion trap conditions.

case of dihydro-MCLR, the MeAla containing fragment ion is shifted by 2 mass units; in the case of MCYR, the Tyr containing fragment ion is shifted by 50 mass units; and in the case of 3-des-MCLR, the Asp containing fragment ion is shifted by -14 mass units. The principal fragment ions of MCLR are compared with those of three related microcystins (Table 1). As discussed above, they are classified into two groups, the shifted or non-shifted groups. This shifting behavior definitely supported the fact that the fragmentation of MCLR and related microcystin using LC/MS/MS under ion trap conditions is reproducible, and these fragment ions are available for sequencing the 
Table 1 Fragment ions of MCLR, dihydro-MCLR, MCYR and 3-des-MCLR observed in the MS² spectra using LC/MS/MS under ion trap conditions.

\begin{tabular}{|c|c|c|c|c|c|}
\hline \multirow{3}{*}{ Ion series } & \multirow{3}{*}{ Ion } & \multicolumn{4}{|c|}{ Microcystins } \\
\hline & & MCLR & Dihydro-MCLR & MCYR & 3-Des-MCLR \\
\hline & & \multicolumn{4}{|c|}{$m / z$} \\
\hline & $\mathrm{M}+\mathrm{H}$ & 995 & $997(+2)$ & $1045(+50)$ & $981(-14)$ \\
\hline \multicolumn{6}{|c|}{ Fragment ion } \\
\hline$a$ & Arg-Adda-Glu-R7-Ala-R2 & 866 & $868(+2)$ & $916(+50)$ & 866 \\
\hline$b$ & Arg-Adda-Glu-R7 & 682 & - & 682 & - \\
\hline$c$ & Arg-Adda-Glu & 599 & 599 & 599 & 599 \\
\hline$d$ & Arg-Adda & 470 & 470 & - & - \\
\hline$e$ & R7-Ala-R2-R3-Arg-Adda & 866 & $868(+2)$ & $916(+50)$ & $852(-14)$ \\
\hline$f^{\prime}$ & R7-Ala-R2-R3-Arg-NH ${ }_{2}$ & 570 & $572(+2)$ & $620(+50)$ & $556(-14)$ \\
\hline$f$ & R7-Ala-R2-R3-Arg & 553 & $555(+2)$ & $603(+50)$ & $539(-14)$ \\
\hline$g$ & R3-Arg-Adda-Glu & 728 & 728 & 728 & $714(-14)$ \\
\hline$g^{\prime}$ & R3-Arg-Adda-Glu(- $\left.\mathrm{H}_{2} \mathrm{O}\right)$ & 710 & 710 & 710 & $696(-14)$ \\
\hline \multirow[t]{5}{*}{$h$} & Ala-R2-R3-Arg & 470 & 470 & $520(+50)$ & $456(-14)$ \\
\hline & & \multicolumn{4}{|c|}{ Constituent amino acid } \\
\hline & $\mathrm{R} 2$ & Leu & Leu & Tyr & Leu \\
\hline & R3 & MeAsp & MeAsp & MeAsp & Asp \\
\hline & $\mathrm{R7}$ & Mdha & MeAla & Mdha & Mdha \\
\hline
\end{tabular}

-: Not observed.

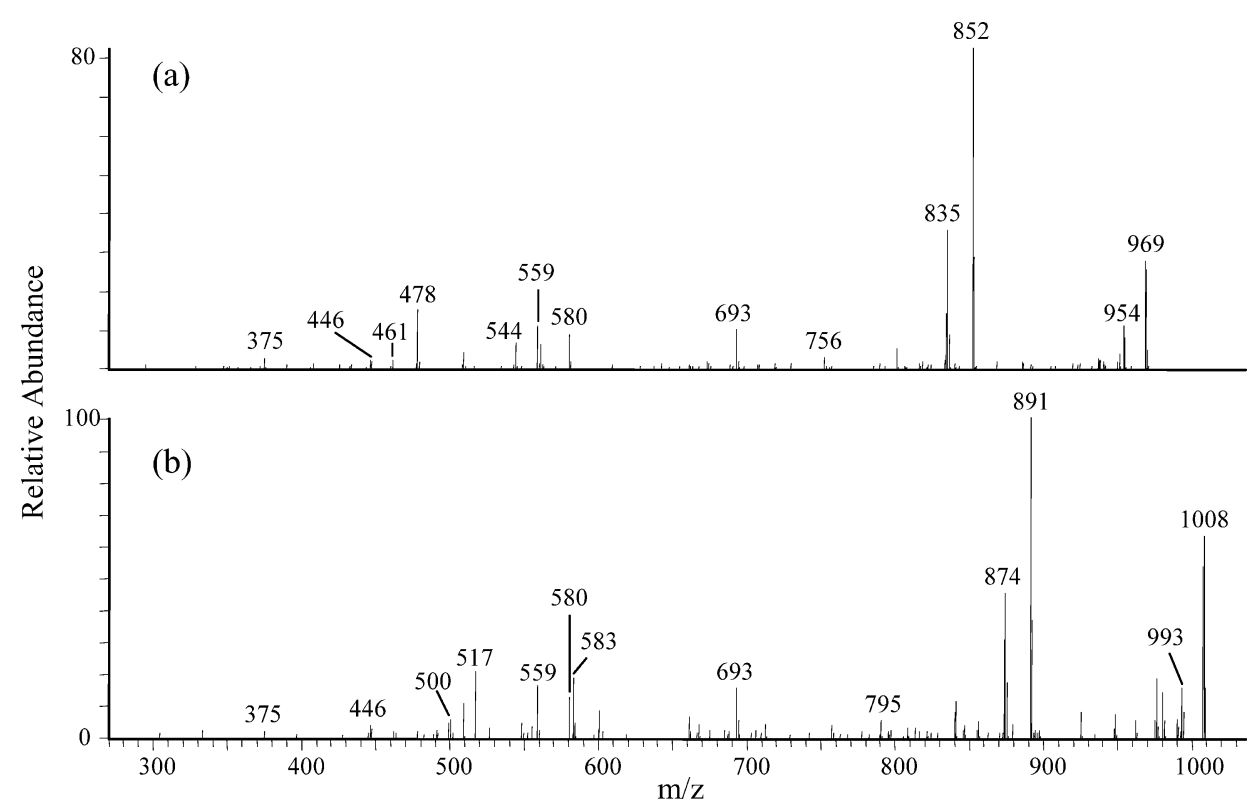

Fig. $4 \mathrm{MS}^{2}$ Spectra of MCLF (a) and MCLW (b). 
constituent amino acids in the microcystins.

\section{Fragmentation Pattern of Microcystin without Arg}

As mentioned above, the mass spectrometric behavior of MCLR and related microcystins was discussed and it was found that any detected fragment ion contained the Arg moiety. It is well known that microcystins without Arg as the constituent amino acid are also produced by cyanobacteria and they often are designated as a "hydrophobic microcystin". Microcystin-LF (MCLF, M.W. 985) and -LW (MCLW, M.W. 1024) contain Phe and Trp instead of Arg in the MCLR, respectively (Fig. 1). The MS ${ }^{2}$ spectra of the protonated molecules for the two microcystins are shown in Fig. 4 and the observed ions are summarized together with those of the MCLR in Table 2. The ion series $f$ and $h$ were only detected in MCLF and MCLW, but almost no other ions were identified as being in the ion series observed in the MCLR and related microcystins. Whereas the fragment ions at $\mathrm{m} / z$ 693, 580, 559, 446 and 375 were commonly observed in the MCLF and MCLW, other ions at $\mathrm{m} / \mathrm{z} 891$ (852), 874 (835) and 795 (756) individually appeared. The fragment ions at $\mathrm{m} / \mathrm{z} 891$ and 852 were found as the base peaks in the mass spectra of the MCLW and MCLF, respectively. These ions were 134 mass units less than the precursor ions $\left((\mathrm{M}+\mathrm{H})^{+}\right)$and considered to be generated by the cleavage between the 8 and 9 positions in

Table 2 Fragment ions of MCLF and MCLW observed in the MS² spectra using LC/MS/MS under ion trap conditions

\begin{tabular}{|c|c|c|c|c|}
\hline \multirow{3}{*}{ Ion series } & \multirow{3}{*}{ Ion } & \multicolumn{3}{|c|}{ Microcystins } \\
\hline & & MCLR & MCLF & MCLW \\
\hline & & \multicolumn{3}{|c|}{$m / z$} \\
\hline & $\mathrm{M}+\mathrm{H}$ & 995 & $986(-9)$ & $1025(+30)$ \\
\hline \multicolumn{5}{|c|}{ Fragment ion } \\
\hline a & R4-Adda-Glu-Mdha-Ala-Leu & 866 & - & - \\
\hline$b$ & R4-Adda-Glu-Mdha & 682 & - & - \\
\hline$c$ & R4-Adda-Glu & 599 & - & - \\
\hline$d$ & R4-Adda & 470 & - & - \\
\hline$e$ & Mdha-Ala-Leu-MeAsp-R4-Adda & 866 & - & - \\
\hline$f^{\prime}$ & Mdha-Ala-Leu-MeAsp-R4-NH 2 & 570 & - & - \\
\hline$f$ & Mdha-Ala-Leu-MeAsp-R4 & 553 & $544(-9)$ & $583(+30)$ \\
\hline$g$ & MeAsp-R4-Adda-Glu & 728 & - & - \\
\hline$g^{\prime}$ & MeAsp-R4-Adda-Glu(- $\left.\mathrm{H}_{2} \mathrm{O}\right)$ & 710 & - & - \\
\hline$h^{\prime}$ & Ala-Leu-MeAsp-R4-NH ${ }_{2}$ & - & 478 & 517 \\
\hline \multirow[t]{11}{*}{$h$} & Ala-Leu-MeAsp-R4 & 470 & $461(-9)$ & $500(+30)$ \\
\hline & Adda-Glu-Mhda-Ala-Leu & - & 693 & 693 \\
\hline & Adda-Glu-Mdha-Ala & - & 580 & 580 \\
\hline & Adda-Glu-Mdha-Ala-Leu-MeAsp-R4(-135) & - & 852 & 891 \\
\hline & Adda-Glu-Mdha-Ala-Leu-MeAsp-R4(-135- $\left.\mathrm{NH}_{3}\right)$ & 844 & 835 & 874 \\
\hline & Adda-Glu-Mdha-Ala-Leu(-135) & - & 559 & 559 \\
\hline & Adda-Glu-Mdha-Ala(-135) & 446 & 446 & 446 \\
\hline & Adda-Glu-Mdha(-135) & 375 & 375 & 375 \\
\hline & MeAsp-R4-Adda-Glu-Mdha-Ala(-135) & - & 756 & 795 \\
\hline & & \multicolumn{3}{|c|}{ Constituent amino acid } \\
\hline & R4 & Arg & Phe & Trp \\
\hline
\end{tabular}

—: Not observed. 


\begin{tabular}{|c|c|c|c|c}
\cline { 2 - 4 } \multicolumn{1}{c|}{} & Glu & \multicolumn{2}{c|}{ Mdha } & \multicolumn{1}{c}{ MCLF } \\
\hline Adda & + & Ala & m/z 986 $(\mathrm{M}+\mathrm{H})^{+}$ \\
\hline Phe & MeAsp & Leu &
\end{tabular}

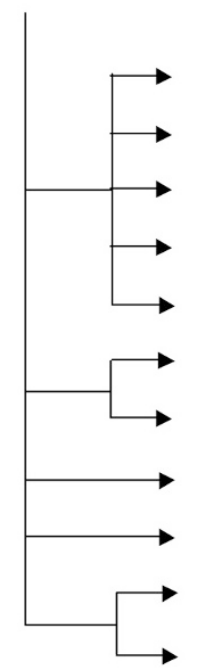

$$
\begin{aligned}
& \text { m/z } 852 ; \text { Adda - Glu - Mdha - Ala - Leu - MeAsp - Phe (- 135) } \\
& \text { m/z } \left.835 ; \text { Adda - Glu - Mdha - Ala - Leu - MeAsp - Phe (- } 135-\mathrm{NH}_{3}\right) \\
& \text { m/z } 559 ; \text { Adda - Glu - Mdha - Ala - Leu (- 135) } \\
& \text { m/z } 446 ; \text { Adda - Glu - Mdha - Ala (- 135) } \\
& \text { m/z } 375 ; \text { Adda - Glu - Mdha (- 135) } \\
& \text { m/z } 693 ; \text { Adda - Glu - Mdha - Ala - Leu } \\
& \text { m/z } 580 ; \text { Adda - Glu - Mdha - Ala } \\
& \text { m/z } 756 ; \text { MeAsp - Phe - Adda - Glu - Mdha - Ala (- 135) } \\
& \text { m/z } 544 ; \text { Mdha - Ala - Leu - MeAsp - Phe } \\
& \text { m/z } 478 ; \text { Ala - Leu - MeAsp - Phe - NH } \\
& \text { m/z } 471 ; \text { Ala - Leu - MeAsp - Phe }
\end{aligned}
$$

Fig. 5 Fragmentation scheme of MCLF using LC/MS/MS under ion trap conditions.

the side chain of Adda. This cleavage has been previously reported [11]. Thus the fragment ions detected in the $\mathrm{MS}^{2}$ spectra of MCLF and MCLW are grouped into three categories: the first one contains the initial carbon-carbon cleavage at the Adda moiety followed by fission of the cyclic structure into a linearized one and subsequent fission of the peptide bonds, the second consists of the fission of the cyclic structure at a peptide bond between Adda and Leu (Trp) into linearized one, but the carbon-carbon cleavage does not occur, and the third one is found in the case of the MCLR. The fragmentation scheme for MCLF is depicted in Fig. 5 and provides informative sequence information even if the carbon-carbon cleavage occurs at the Adda moiety.

\section{Structural Characterization of Microcystins in Cyanobacterial Blooms by LC/MS}

The systematic study mentioned above strongly suggested that the fragment ions of microcystins generated in the $\mathrm{MS}^{2}$ analysis of the LC/MS/MS under ion trap conditions are informative and available for the sequencing the constituent amino acids. Therefore, the established fragmentation pattern was applied to identify the microcystins in the crude extracts from a bloom sample. The total ion chromatography (TIC) and mass chromatograms of the microcystins detected in the LC/MS/MS under ion trap conditions of the crude extracts from the lyophilized cells of cyanobacteria collected from Lake Suwa in August 2005 are shown in Fig. 6. Four ion peaks were readily identified as MCLR and 7-des-MCLR, MCRR, MCYR based on their molecular weights and retention times. However, it was difficult to identify two peaks eluted at 16.65 minutes (peak A) and 18.61 minutes (peak B) using only the information concerning their molecular weights and retention times. Peak A showed the protonated molecule at $m / z$ 1049, which is 4 mass units higher than MCYR. The MS ${ }^{2}$ spectrum of peak A is shown in Fig. 7, in which the prominent fragment ions were observed at $\mathrm{m} / \mathrm{z} 920,728,710,686,624,607$ and 599 in the $\mathrm{MS}^{2}$ analysis of the protonated molecule at $\mathrm{m} / \mathrm{z}$ 1049 as the precursor ion. The resulting fragmentation pattern showed that peak A contains Arg. The fragment ions at $\mathrm{m} / \mathrm{z}$ 728, 710 and 599 were commonly observed in the spectrum of the MCYR, and identified as Ion $g, g^{\prime}$ and c. Four fragment ions at $m / z$ 920, 686, 624 and 607 appeared 4 mass units higher than Ions $a$ or $e, b, f^{\prime}$ and $f$ of the MCYR, respectively. Thus, a linear structure, TyrMeAsp-Arg-Adda-Glu, was determined as the partial structure of peak A. A fragment ion at $\mathrm{m} / \mathrm{z} 920$ was detected 129 mass units less than the protonated molecule at $m / z 1049$, and this difference corresponded to loss of Glu or MeAsp. This indicated that the structural variation between peak A and MCYR is due to the Mdha or Ala moiety. The ion at $m / z 686$ was 4 mass units higher than $\mathrm{m} / \mathrm{z} 682$ (Arg-Adda-Glu-Mdha) observed in the $\mathrm{MS}^{2}$ analysis of the MCYR, suggesting that this fragment ion was Arg-Adda-Glu-Ser. Therefore, peak A was considered to be $\left[\mathrm{Ser}^{7}\right]$-MCYR, in which Mdha in the MCYR was replaced by Ser and the predicted fragmentation pattern for 


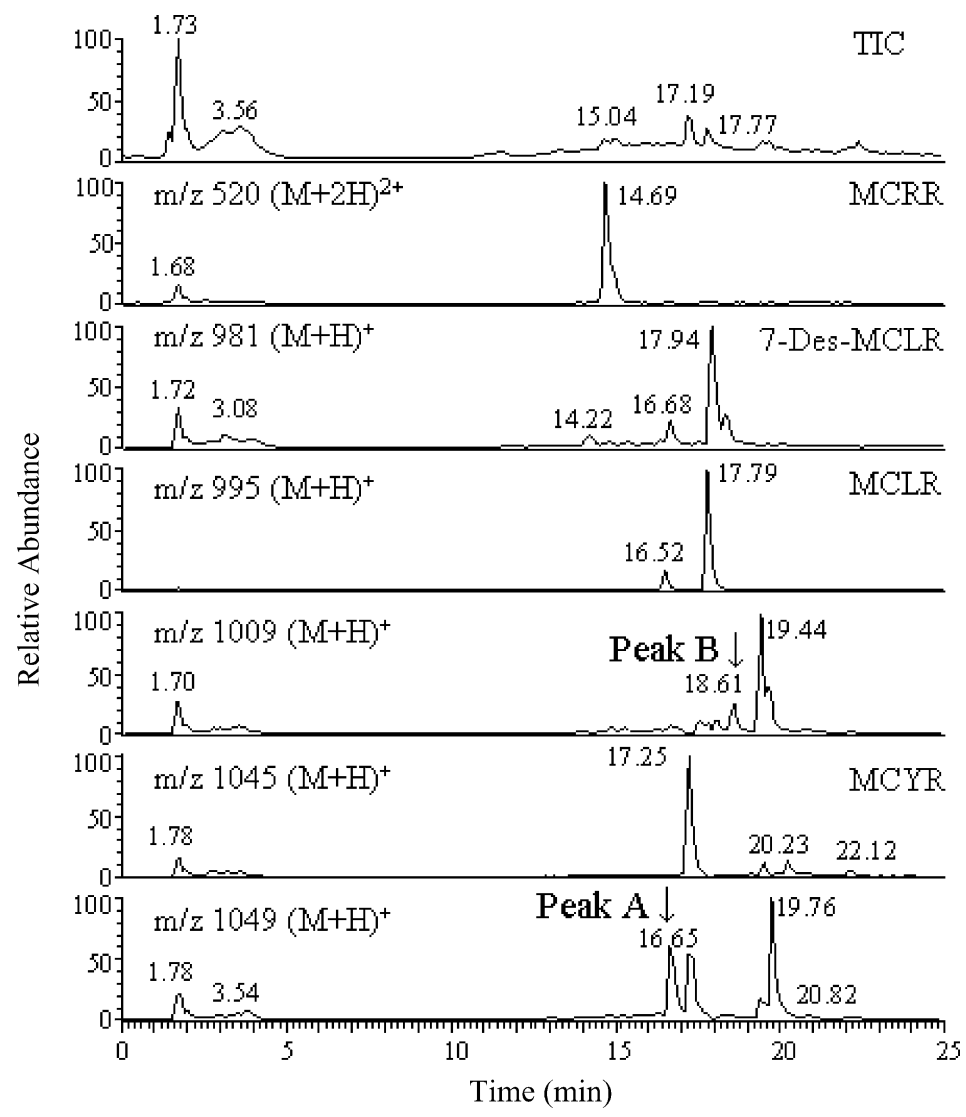

Fig. 6 Total ion chromatogram and mass chromatograms of microcystins of bloom extracts collected from Lake Suwa.

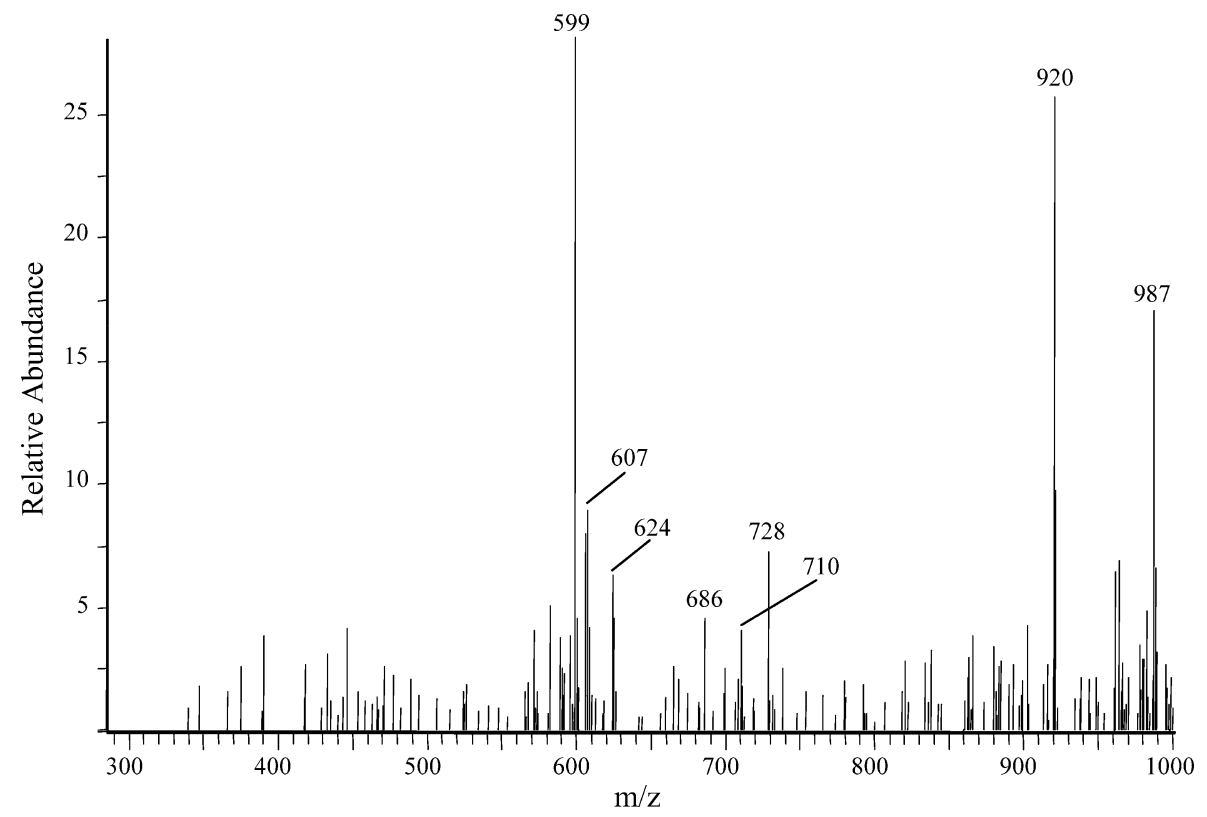

Fig. $7 \mathrm{MS}^{2}$ spectrum of Peak A. 
peak A coincide with the established one. Thus, peak A was identified to $\left[\mathrm{Ser}^{7}\right] \mathrm{MCYR}$ apart from the absolute configuration of Ser.

Peak B showed the protonated molecule at $\mathrm{m} / \mathrm{z} 1009$ in the LC/MS/MS under ion trap conditions. The fragment ions were observed at $\mathrm{m} / \mathrm{z} 880,710,682,599,584,567$ and 484 in the $\mathrm{MS}^{2}$ analysis of the protonated molecule at $\mathrm{m} / \mathrm{z}$ 1009 as the precursor ion (data not shown). The fragment ions at $\mathrm{m} / \mathrm{z} 710$ and 599 were identified as Ions $\mathrm{g}^{\prime}$ and $c$, respectively. Because the fragment ion at $\mathrm{m} / \mathrm{z} 682$ corresponded to Ion $b$, a partial structure of peak B was supposed to be MeAsp-Arg-Adda-Glu-Mdha. Other fragment ions at $m / z 880,584,567$ and 484 were 14 mass units higher than Ions $a$ or $e, f^{\prime}, f$ and $h$. These data suggested that peak B contains the methylated Ala or Leu as the constituent amino acid, so that the structure was considered to be [methylated $\mathrm{Ala}^{1}$ ] or [methylated Leu ${ }^{2}$ ] MCLR. Such a microcystin has not yet been reported.

\section{Discussion}

Many cyclic peptides are used in modern life and possibly in our future. As discussed in the introduction, no suitable methodology has been developed for the structural characterization of cyclic peptides, because cyclic peptides from natural sources have complicated structures including unusual amino acids. Particularly, no reproducible and reliable method has been established for the sequencing of the constituent amino acids in the case of a small amount of sample. Although tandem mass spectrometry, such as four sector MS/MS and triple stage MS/MS, has been used for this purpose, they have not provided satisfactory results [11 14]. In this study, a systematic investigation was planned to confirm the applicability of LC/MS/MS under ion trap conditions for the sequencing using microcystin as a typical cyclic peptide, and the final goal was to successfully apply the obtained information of known microcystins to characterize unknown ones.

First of all, the mass spectrometric behavior of the microcystin was carefully examined under $\mathrm{MS}^{2}$ analysis conditions. The $\mathrm{MS}^{2}$ spectrum of the protonated molecule $(\mathrm{M}+\mathrm{H})^{+}$provided many fragment ions which are mainly generated by the cleavage of peptide bonds. These ions were formed by the two-bond fissions of the precursor ion, in which an initial fission of the cyclic structure to a linearized one and subsequent fission(s) at the peptide bonds are included. They belong to the $b n$ ions according to the notation by Biemann [24], which are desirable for the sequencing. In the case of the microcystin-containing Arg, the observed ions always contain the Arg moiety (Fig. 3).
The fragmentation of other microcystins without Arg showed a different pattern and the cleavage between the 8 and 9 positions in the side chain of Adda predominates prior to the fission of the peptide bonds (Fig. 5). The obtained fragmentation was confirmed by a shifting technique using structurally related microcystins. Diehnelt et al. reported the identification of the fragment ions of microcystins using an ion tap Fourier transform ion cyclotron [10]. They also analyzed MCLF and the fragment ions at $m / z 852,835,693,580,559$ and 544 were similar to the fragment ions obtained from our experiment, which are available for the sequence information. In other microcystins, the fragment ions were identified by their $\mathrm{m} / \mathrm{z}$ value and molecular composition, and most of them coincided with our proposed fragment ions. Moreover, they reported that the fragmentation of microcystin containing Arg or without Arg showed similarly the different pattern. These results indicate that fragment ions obtained from LC/MS/MS under ion trap conditions are reproducible and this system is applicable to the structural characterization of microcystins. Poon et al. also reported the analysis of microcystins using LC/MS/MS under ion trap conditions, and the fragment ions obtained from the $\mathrm{MS}^{2}$ analysis was described in detail [18]. However, their proposed fragmentation pattern was not systematically discussed, and it was difficult to apply them for the structural characterization of unknown microcystins, because there was no discussion regarding the generation of fragment ions including the two-bond fission and the difference of fragmentation pattern of microcystins containing Arg or without Arg as discussed above.

The established fragmentation pattern was then used to identify the microcystins in the crude extracts from a bloom sample, and 4 known and 2 unknown microcystins were found. One of the unknown microcystins was identified to [Ser $\left.{ }^{7}\right]$ MCYR, but another corresponded to either the [methylated $\left.\mathrm{Ala}^{1}\right]$ or [methylated $\mathrm{Leu}^{2}$ ] MCLR. The structure was not fully elucidated, because there were no informative ions generated by the cleavage of the peptide bond between Ala and Leu. We considered that this method is difficult to apply to the structural characterization of unknown microcystins structurally modified at $\left[\mathrm{Ala}^{1}\right]$ or $\left[\mathrm{R}_{4}\right]$ position (Fig. 1). $\mathrm{MS}^{2}$ spectrum using centroid mode was not always sufficient because obtained data was not scanned in profile mode. However we have analyzed isolated microcystins in centroid mode and made sure that the initial fission occurred the peptide bond definitely and fragment ions were generated as $b_{n}$ series ions. Further, we could find the fragmentation pattern of microcystins containing Arg or without Arg and clarified the difference. Therefore the obtained fragment ions using centroid mode 
was applicable to the structural characterization of unknown microcystins and make possible to distinguish the fragment ion peaks from noise peaks. These experiments shown in this study strongly indicated that the obtained fragmentation pattern of the microcystins by LC/MS/MS under ion trap conditions is reproducible, and these fragment ions are available for sequencing the constituent amino acids in the microcystins. In a subsequent step, we have to confirm that the established fragmentation by $\mathrm{LC} / \mathrm{MS} / \mathrm{MS}$ under ion trap conditions is available or not for other types of cyclic peptides.

Acknowledgement We would like to thank Dr. Jussi Meriluoto (Abo Akademi University) for providing MCLF and MCLW.

References

1. Lockhart IM, Newton GG, Abraham EP. Structure of bacitracin A. Nature 173: 536-537 (1954)

2. Suzuki T, Hayashi K, Fujikawa K. Studies on the chemical structure of collistin. III. Enzymatic hydrolysis of colistin A. J Biochem (Tokyo) 54: 412-418 (1963)

3. Smith KA, Williams DH, Smith GA. Structure studies on antibiotic vancomycin; the nature of the aromatic rings. J Chem Soc Perkin Trans I 20: 2369-2376 (1974)

4. Tomishima M, Ohki H, Yamada A, Takasugi H, Maki K, Tawara S, Tanaka H. FK463, a novel water-soluble echinocandin lipopeptide: synthesis and antifungal activity. J Antibiot (Tokyo) 52: 674-676 (1999)

5. Petcher TJ, Weber H, Ruegger A. Crystal and molecular structure of an iodo-derivative of the cyclic undecapeptide cyclosporin A. Helv Chim Acta 59: 1480-1489 (1976)

6. Borel JF, Padova FDI, Mason J, Quesniaux V, Ryffel B, Wegner R. Pharmacology of cyclosporin (Sandimmune) I. Introduction. Pharmacol Rev 41: 239-242 (1989)

7. Schepdael AV, Govaerts C, Li C, Orwa J, Adams E, Roets E, Hoogmartens J. Sequencing of bacitracin A and related minor components by liquid chromatography/electrospray ionization ion trap tandem mass spectrometry. Rapid Commun Mass Spectrom 17: 1366-1379 (2003)

8. Mackintosh C, Beattie KA, Klumpp S, Cohen P. Codd GA. Cyanobacterial microcystin-LR is a potent and specific inhibitor of protein phosphatases 1 and $2 \mathrm{~A}$ from both mammals and higher plants. FEBS Lett 264: 187-192 (1990)

9. Botes DP, Tuinman AA, Wessels PL, Viljoen CC, Kluger H, Williams DH, Santikarn S, Smith RJ, Hammond SJ. The structure of cyanoginosin-LA, a cyclic heptapeptide toxin from the cyanobacterium Microcystis aeruginosa. J Chem Soc Perkin Trans I 2311-2318 (1984)

10. Budde WL, Diehnelt CW, Dugan NR, Peterman SM. Identification of microcystin toxins from a strain of Microcystis aeruginosa by liquid chromatography introduction into a hybrid linear ion trap-fourier transform ion cyclotron resonance mass spectrometer. Anal Chem 78:
501-512 (2006)

11. Namikoshi M, Rinehart KL, Sakai R, Stotts RR, Dahlem AM, Beasley VR, Carmichael WW, Evans WR. Identification of 12 hepatotoxins from a homer Lake broom of the cyanobacteria Microcystis aeruginosa, Microcystis viridis, and Microcystis wesenbergii: Nine New Microcystins. J Org Chem 57: 866-872 (1992)

12. Rinehart KL, Namikoshi M, Choi BW. Structure and biosynthesis of toxins from blue-green algae (cyanobacteria). J Appl Phycol 6: 159-176 (1994)

13. Yuan M, Namikoshi M, Otsuki A, Rinehart KL, Sivonen K, Watanabe MF. Low-energy collisionally activated decomposition and structural characterization of cyclic heptapeptide microcystins by electrospray ionization mass spectrometry. J Mass Spectrom 34: 33-43 (1999)

14. Kubwabo C, Vais N, Benoit FM. Characterization of microcystins using in-source collision-induced dissociation. Rapid Commun Mass Spectrom 19: 597-604 (2005)

15. Schwartz JC, Jardine I. Quadrupole ion trap mass spectrometry. Methods Enzymol 270: 552-586 (1996)

16. Elias JE, Haas W, Faherty BK, Gygi SP. Comparative evaluation of mass spectrometry platforms used in largescale proteomics investigations. Nat Methods 2: 667-675 (2005)

17. Kostiainen R, Kotianho T, Kuuranne T, Auriola S. Liquid chromatography/atomospheric pressure ionization-mass spectrometry in drug metabolism studies. J Mass Spectrom 38: 357-372 (2003)

18. Zweigenbaum JA, Henion JD, Beattie KA, Codd GA, Poon GK. Direct analysis of microcystins by microbore liquid chromatography electrospray ionization ion-trap tandem mass spectrometry. J Pharm Biomed Anal 23: 724-733 (2000)

19. Harada K, Matsuura K, Suzuki M, Oka H, Watanabe MF, Oishi S, Dahlem AM, Beasley VR, Carmichael WW. Analysis and purification of toxic peptides from cyanobacteria by reversed-phase high-performance liquid chromatography. J Chromatogr 448: 275-283 (1988)

20. Harada K, Suzuki M, Dahlem AM, Beasley VR, Carmichael WW, Rinehart KL Jr. Improved method for purification of toxic peptides produced by cyanobacteria. Toxicon 26: 433-439 (1988)

21. Namikoshi M, Choi BW, Sun F, Rinehart KL, Evans WR, Carmichael WW. Chemical characterization and toxicity of dihydro derivatives of nodularin and microcystin-LR, potent cyanobacterial cyclic peptide hepatotoxins. Chem Res Toxicol 6: 151-158 (1993)

22. Rinehart KL, Harada K, Namikoshi M, Chen C, Harvis CA, Munro MHG, Blunt JW, Mulligan PE, Beasley VR, Dahlem AM, Carmichael WW. Nodularin, microcystin, and the configuration of Adda. J Am Chem Soc 110: 8557-8558 (1988)

23. McLafferty FW, Interpretation of Mass Spectra, University Science Books: 100-101 (1980)

24. Biemann K. Contributions of mass spectrometry to peptide and protein structure. Biomed Environ Mass Spectrom 16: 99-111 (1988) 